\title{
Harnessing the Natural Toxic Metabolites in COVID-19
}

\author{
Ali Bahrami $\mathbb{D},,^{1,2}$ Mohammad Taheri $\mathbb{D},{ }^{3}$ Mohammad Reza Arabestani $\mathbb{D},^{3}$ \\ Meysam Soleimani $\mathbb{D}^{2},{ }^{2}$ Mojdeh Mohammadi $\left(\mathbb{D},{ }^{4}\right.$ Fatemeh Golabchi, ${ }^{1,2}$ \\ Maryam Banitorfi, ${ }^{1,2}$ Seyed Mostafa Hosseini $\mathbb{D}^{3}{ }^{3}$ Sodabe Khodabandehlou, ${ }^{4}$ \\ and Fatemeh Nouri (iD ${ }^{2}$ \\ ${ }^{1}$ Student Research Committee, Hamadan University of Medical Sciences, Hamadan, Iran
${ }^{2}$ Department of Pharmaceutical Biotechnology, School of Pharmacy, Hamadan University of Medical Scienceaffs, Hamadan, Iran
${ }^{3}$ Department of Microbiology, Faculty of Medicine, Hamadan University of Medical Sciences, Hamadan, Iran
${ }^{4}$ Department of Pharmacology and Toxicology, School of Pharmacy, Hamadan University of Medical Sciences, Hamadan, Iran
}

Correspondence should be addressed to Mohammad Taheri; motaheri360@gmail.com and Fatemeh Nouri; fatemenouri1@gmail.com

Received 6 November 2021; Revised 16 January 2022; Accepted 9 February 2022; Published 7 March 2022

Academic Editor: Jelena Zivkovic

Copyright ( $\odot 2022$ Ali Bahrami et al. This is an open access article distributed under the Creative Commons Attribution License, which permits unrestricted use, distribution, and reproduction in any medium, provided the original work is properly cited.

SARS-CoV-2 is a novel coronavirus and the cause of the recent pandemic; it is an enveloped $\beta$-coronavirus. SARS-CoV-2 appear in the Wuhan City of China for the first time and outspread worldwide quickly. Due to its person-to-person fast transmission, COVID-19 is becoming a global problem. SARS-CoV-2 enter into cells by using ACE2 receptors that are numerous in the lungs and finally can cause acute respiratory distress syndrome (ARDS). Dry cough, sore throat, fever, body pain, headache, GIT discomfort, diarrhoea, and fatigue are some of the COVID-19 symptoms. There is no definite and certain treatment for disease caused by SARS-CoV-2 till now. Some pharmacological effects of toxins, toxoids, and venoms have been proven, and their effects on some diseases have been evaluated. This study aimed to investigate the role of toxins, toxoids, and venom in the pathophysiology of COVID-19 disease.

\section{Background}

Acute respiratory syndrome coronavirus 2 (SARS-CoV-2) is characterized by severe cytokine syndrome following inflammation. The pathogenesis of SARS-CoV-2 is such that in the first stage, viral binding to epithelial cells occurs with a minimal innate immune response. In the second stage, we see increased viral replication along with an active immune response and the spread of the virus to the lower respiratory tract, which may also affect the digestive and cardiovascular systems. In the third stage, events such as hypoxia, the penetration of the virus into the entire respiratory tract, and finally acute respiratory distress syndrome (ARDS) occur, which the fatal rate is potentially high [1].

SARS-CoV-2 affects the lungs the most, but it can also attack the brain and cause unpredictable nervous defects by crossing the blood-brain barrier [2]. SARS-CoV-2 is transmitted person-to-person through viral airborne droplets. This infection has several clinical symptoms such as dry cough, sore throat, fever, body pain, headache, abdominal discomfort, diarrhoea, and fatigue. In the acute phase, it can cause pneumonia, acute respiratory failure, encephalopathy, multiorgan dysfunction, and death. Because angiotensin-converting enzyme 2 (ACE2) is one of the major receptors identified for SARS-CoV-2 and is predominantly expressed in the lungs, the lungs are involved in coronavirus infection. This receptor can also be found in the gastrointestinal tract, the cardiovascular system, the brain, and other organs [3-5].

Various data and treatments for SARS-CoV-2 infection are being reviewed. We previously examined the impact of serum electrolytes, trace elements, and heavy metals in COVID-19 [6, 7]. Toxins are small molecules, peptides, or proteins secreted by prokaryotic and eukaryotic cells and provide defence ability for them [8]. Phospholipases, proteases, and ion channel modulators are their primary targets. 
Many toxins are multifunctional and have several biological targets which may have no relation with their toxic role [9]. Some toxin-derived peptides are now being used to treat type 2 diabetes, hypertension, neuropathic pain, and other medical disorders [10-13]. Some data confirm the effect of bee venom (BV) on preventing COVID-19 and improving it [1]. Some other data ignore the BV effect on preventing COVID-19 and hypothesize that less SARS-CoV-2 infection in beekeepers is due to their less exposure to other people [3]. A low dose of botulinum neurotoxin (BoNT) can reduce the symptoms of COVID-19, and so, it could be used in treatment lines [14]. It has demonstrated that the rate of DTP vaccination has an inverse correlation with COVID-19 prevalence [15]. Cobrotoxin has an anti-inflammatory effect and also can restore the CD4/CD8 ratio and perform immunoprotective activity against SARS-CoV-2 [16]. Tetrodotoxin is an inhibitor of $\mathrm{M}^{\text {Pro }}$ of SARS-CoV-2 and so can affect the virus [17]. This study aimed to investigate the role of toxins, toxoids, and venom in the pathophysiology of COVID-19 disease.

\section{Method}

The bibliographic search was performed on PubMed, Scopus, and Web of Science databases in Sep 2021. Search keywords including "toxin" OR "aflatoxin" OR "botulinum toxin A" OR “dioxin" OR "diphtheria toxin" OR "exotoxin” OR "pertussis toxin" OR "tetanus toxin" OR "tetrodotoxin" OR "trichothecenes" OR "Shiga toxin" OR "cholera toxin B" OR "bufotoxin" OR "ochratoxin A" OR “Anthrax toxin" AND “coronavirus 2019" OR “COVID-19” OR "SARS-CoV2 " in all fields. Any languages or date restrictions were not applied. Identified studies were screened by title, abstract, and full text. The reference list of identified studies was also evaluated to increment the sensitivity and choice of most literature which we could not identify in the database. Greatest sensitivity search was initiated, 167 articles on external databases were found and collected by a researcher using the Endnote software. Then, unifying the articles from all the cited databases and bringing out duplicate articles, the two researchers separately investigated all the articles and excluded the articles that includes irrelevance of title to toxins and COVID-19, absence of keywords in the title or abstract of studies, and the inclusion index criteria. Afterwards, several articles after reviewing titles and abstracts were excluded. The existing publications were carefully assessed, the relevant study was chosen, and the data for the studies were appraised based on the title, examined technique, sample size, and so on. Finally, 38 articles were analyzed after acquiring relevant papers and determining the limitations of the search strategy. During the examination of publications in 2021, we discovered 30 new articles that were included in the study (search strategy was shown in Figure 1).

\section{Results}

3.1. Bee Venom. Apitherapy means the use of bee stings to treat certain diseases. However, the results regarding the effectiveness of this method are controversial [3]. Bee venom

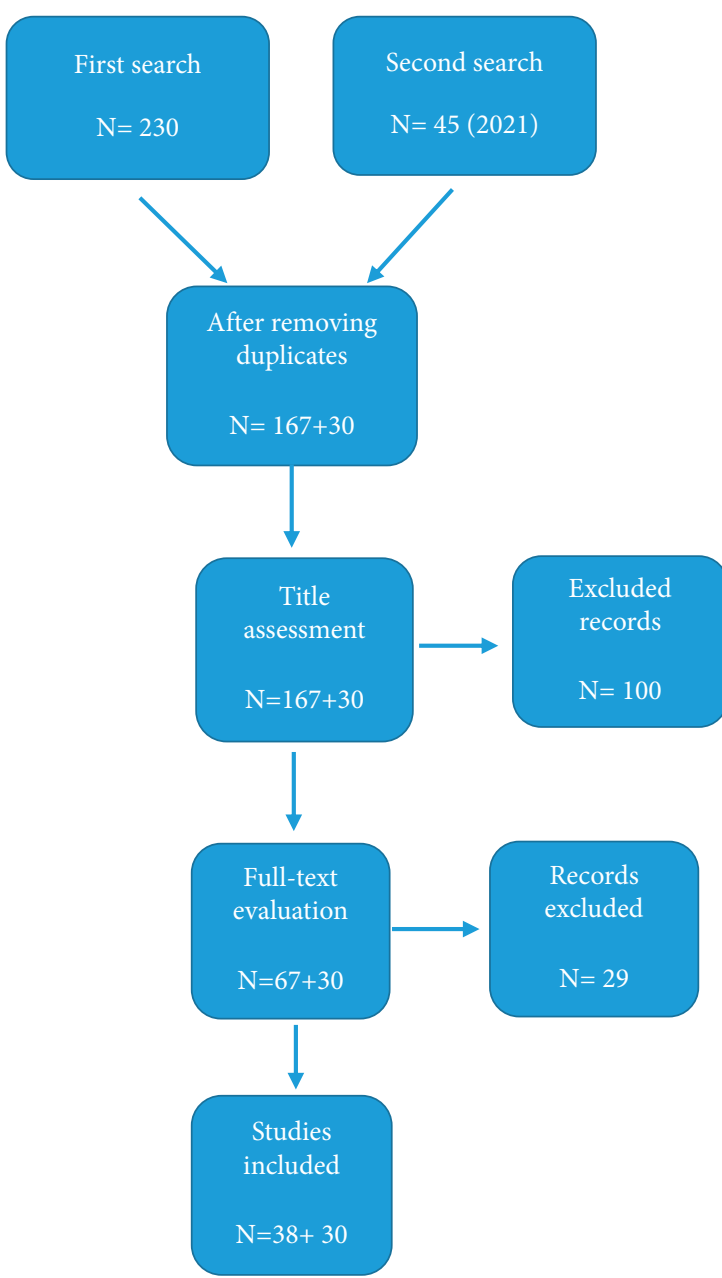

FIGURE 1: Flow chart of the search strategy.

(BV) has antiviral properties because of the presence of melittin and phospholipase A2 (PLA2). Both agents work against enveloped and nonenveloped viruses, and they work against $\mathrm{H} 1 \mathrm{~N} 1$ and $\mathrm{HIV}$ by using antagonist activity against IL-6, IL-8, interferon, and TNF- $\alpha$. BV vaccination is also a way to protect against cytomegalovirus. In modern medicine, bee venom is used to treat Parkinson's disease, multiple sclerosis, and arthritis [1, 18-20].

According to a study in Hubei Province in China, none of the 5115 local beekeepers had covid-19 symptoms. 5 apitherapists and 121 patients who received apitherapy were also interviewed. Two of the apitherapists, although exposed to suspected or confirmed covid-19 victims without protection, showed no signs of COVID-19. Also, none of the 121 patients was infected with COVID-19, even though 3 of them were exposed to relatives infected with SARS-CoV-2. They suggested that BV therapy, due to the presence of melittin and phospholipase A2, which have a strong antiinflammatory function, could help support recovery because even if the patient recovers from the initial SARS-CoV-2 infection, and it may have long-term effects which are known as long-covid because PLA2 binds to the membrane in vivo, causes antibodies to bind with the cell membrane, and stimulates CD8 T cells [1]. Bee venom increases the 
differentiation of FOXP3-expressing cells in CD4 T cells and mature CD4 thymocytes [21, 22]. Yang et al. believe that the effect of BV on the immune system and increasing the differentiation of human regulatory $\mathrm{T}$ cells plays an important role in controlling COVID-19 [18]. Also, Block et al. believe that the anti-inflammatory and antimicrobial properties of BV derivatives may help prevent long-term fibrotic destruction of the lungs. It has demonstrated that IL10 increase in beekeepers due to their persistent exposure to BV. IL-10 is an anti-inflammatory cytokine that reduces inflammatory cytokines IL-1 and TNF-alpha and so, BV can decrease the cytokine storm $[3,23]$.

A study conducted in Germany did not confirm the results of apitherapy in SARS-CoV-2. The study found that beekeepers were not immune to SARS-CoV-2 infections. The hypothesis that beekeepers will not be infected with SARS because of immunity due to bee stings is not supported by the data of this study. Some factors like how long they had been a beekeeper, the total number of bee stings received, the number of bee stings received in the year 2020, and potential allergic reactions to bee stings do not have any effect on the severity of COVID-19. However, beekeepers' reactions to bee stings are one of the elements that influence two COVID-19 symptoms, including tiredness and sore throat soreness. These symptoms are more pronounced in beekeepers who are more sensitive to bee stings. The fact that beekeepers are less affected by SARS-CoV-2 infection may be due to their personality traits, which tend to spend more time alone and therefore less exposed to contact with other humans and COVID-19. BV and the melittin it contains can regulate Th1 and other immune cells and are used to lower viral load and reduce the severity of interstitial pneumonia in PRRSV-infected pigs. These effects may also be crucial in the case of SARS-CoV-2 pneumonia, but these effects were only achieved when BV was administered through the nasal or rectal route. The various effects of $\mathrm{BV}$ on the immune system and its resistance to SARS-CoV- 2 should be considered as an indicator of the level of the immune response, rather than as a definite therapy strategy [3].

3.2. Botulinum Neurotoxin (BoNT). The use of therapeutic botulinum neurotoxin (BoNT) against SARS-CoV-2 is also being considered. Botulinum toxins are powerful neurotoxins that can cause muscle paralysis and acute respiratory arrest in humans, but a mild dose of the pure form of Botox therapy is known to reduce the common clinical symptoms of COVID-19 including chronic cough, dyspnoea, pneumonia, acute respiratory failure, abnormal circulation, cardiac defects, and various neurological deficits. A low dose of purified BoNT is also used in many diseases such as strabismus, blepharospasm, chronic migraine, and overactive bladder. Therapeutic BoNT can be used as a method of preventing SARS-CoV-2 infection in high-risk populations. To reduce its side effects, antioxidants can also be used along with treatment. It reported that the side effects of therapeutic BoNT are temporary and reversible, and the action of this toxin in very mild doses is relatively safe [24]. There are some reports that therapeutic BoNT in very mild doses can suppress many human diseases by 10 to 20 units [25-28]. It also can migrate from the site of intramuscular injection to the brain and other organs. The beneficial effects of a single therapeutic dose are probably long-lasting. Designing a multifaceted drug to fight SARS-CoV-2 infection can be effective, and therapeutic BoNT can be a good option [14]. In a study in France, 193 patients who used BoNT/A in different doses for different purposes such as migraines and facial palsy were evaluated, and a critical contrast between the number of infected people within the common populace and the number of patients injected with BoNT/A who appeared signs of COVID-19 has seen [29].

3.3. Tetanus and Diphtheria. There is evidence suggesting that a tetanus toxoid vaccine could reduce the severity of COVID-19 symptoms. A connection between tetanus toxin and the COVID-19 spike glycoprotein as well as a similar connection between the tetanus toxin protein sequence and other coronaviruses was observed [30]. Also, neurological symptoms and temporomandibular joint (TMJ) pain, which are common symptoms of tetanus disease, have been reported in several COVID-19 patients. Also, in comparison between the United States and other countries where tetanus vaccination rates were lower, an inverse correlation was observed between tetanus vaccination rates and mortality rates [31, 32]. We know the DTP vaccine contains toxoids of tetanus, diphtheria, and pertussis, and it is noteworthy that there is a correlation between the rate of DTP vaccination in children worldwide and the rate of asymptomatic COVID19. A similar association is observed in pregnant women, who are advised to receive two flu and TDaP vaccines in the third month of pregnancy [30]. As a result, it may be inferred that tetanus and diphtheria vaccines may have a protective effect against COVID-19 in both children as well as adults who are up-to-date on their tetanus vaccination. Two other studies also noted a reduction in the severity of COVID-19 in children and its possible association with DTP vaccination and suggested that the DTP vaccine could stimulate the immune system. In one case, it was concluded that the DTP vaccine is the only vaccine with a high potential for crossreactivity with COVID-19 spike protein [15, 33]. CRM197, a modified diphtheria toxin found in the Hib vaccine, and rubella vaccine are highly similar to SARS-CoV-2 proteins, suggesting that they may have some anti-SARS-CoV-2 activity [34].

3.4. Cobrotoxin. Cobrotoxin, a short-chain $\alpha$-neurotoxin from Naja naja atra venom (NNAV), may be effective in treating COVID-19 patients and inhibiting SARS-CoV-2 infection. The inflammatory cytokine storm causes aggravation of lung disease in COVID-19 patients, in addition to other deadly consequences. As a result, in addition to attempting to restrict virus replication, anti-inflammatory medication is an important method of combating COVID19 disease. Cobrotoxin and alpha-neurotoxins have antiinflammatory activity and prevent the binding of the nuclear factor- $\kappa \mathrm{B}$ to DNA, which is a transcription factor that regulates the expression of genes involved in the 
TABLE 1: Data summary.

Bee venom
Botulinum neurotoxin
(BoNT)
Tetanus and diphtheria
Cobrotoxin
Tetrodotoxin

IL-1 and TNF- $\alpha$ decrease because of an increase in cytokine IL-10 due to bee venom, so, cytokines storm could be weakened. Also, bee venom contains melittin and phospholipase A2 that have an antagonist effect on IL-6, IL-8, interferon, and TNF- $\alpha$. $[1,3,18-20,23]$. Less infection in bee keepers is due to their less exposure to other people not because of bee venom [3].

A mild dose of the pure BoNT can reduce the common clinical symptoms of COVID-19 including chronic cough, dyspnoea, pneumonia, acute respiratory failure, abnormal circulation, cardiac defects, and various neurological deficits [14].

Tetanus toxin and diphtheria toxin both are similar to SARS-CoV-2 proteins, and so, the administration of the DTP vaccine may have a protective effect against SARS-CoV-2 [30, 34].

Cobrotoxin prevents the expression of inflammatory genes and also balances the ratio of CD4/CD8 cells and performs immunoprotective activity [16].

Tetrodotoxin is a potential $\mathrm{M}^{\text {Pro }}$ inhibitor of SARS-CoV-2. So, it may affect SARS-CoV-2 [17]. inflammatory response, thereby reducing the transcription of inflammatory genes. Cobrotoxin inhibits CD8 T cell proliferation more than that of CD4 T cells, and since COVID-19 cellular immune responses are induced by overexpression and hyperactivation of cytotoxic T lymphocytes [35], cobrotoxin can restore the CD4/CD8 ratio and perform an immunoprotective activity [16].

3.5. Tetrodotoxin. Tetrodotoxin is a sodium channel blocker and acts on the nervous system message delivery [36]. The toxicity of tetrodotoxin is 1200 times more than cyanide. One of the important drug targets in coronaviruses is the main protease $\left(\mathrm{M}^{\text {Pro }}\right)$ due to its important role in controlling replicate complex activity and its vital role in viral replication and transcription $[37,38]$. Tetrodotoxin is a potential $\mathrm{M}^{\text {Pro }}$ inhibitor of SARS-CoV-2 which may be a potential compound against SARS-CoV-2 according to the results of the ligand-based approaches [17].

3.6. Snake Venoms. Nature is known to offer numerous biotherapeutics from animal venoms, green growth, and plant that have been generally utilized in conventional medication. Among these bioresources, snake poison shows numerous bioactivities such as antiviral, antiplatelet, antithrombotic, anti-inflammatory, antimicrobial, and antitumoral. Snake venom contains a mixture of amino acids, proteins, peptides, nucleotides, lipids, carbohydrates, and metal elements along with proteins $[39,40]$. Several studies have reported that snake venom ingredients have antiviral activity against measles, Sendai, dengue, yellow fever, and human immunodeficiency virus [41-43]. Snake venoms are a complex combination of proteins and peptides. Venom serine proteases (SVSPs), snake venom metalloproteinases (SVMPs), secreted phospholipases A2 (SV-PLA2s), C-type lectins, and disintegrins are the major groups of snake venom components, while the minor group includes nucleotidases (Ntases), phosphodiesterases (PDEs), cysteinerich secretory proteins, L-amino acid oxidases, Kunitz peptides, three-finger peptides (3FTX), and natriuretic peptides [44-48]. Bradykinin-potentiating peptide 10C (BPP-10C) isolated from Bothrops jararaca can decrease angiotensin II by inhibiting the ACE and increase bradykinin 2-receptor [49-51] that both have a role in the pathogenesis of SARS-CoV-2 [52-55], and it can be considered as an anti-SARS-CoV-2 agent [56]. Kunitz-type peptides ((also called bovine pancreatic trypsin inhibitors) (BPTIs)) are 50 to 60 amino acid components found in the snake venoms that inhibit the catalytic site of serine proteases [57]; so, they are potential antiviral agents because transmembrane protease serine-2 (TMPRSS2) activity is required for SARS-CoV-2 entry [58, 59]. Clinical information has appeared that numerous patients with serious COVID-19 display coagulation anomalies such as microvascular thrombosis and venous or blood vessel thrombosis [60-63]. Snake venoms are wealthy sources of bioactive particles interfering with the blood coagulation cascade and platelet aggregation $[64,65]$. Phospholipase A2s (PLA2s) are one of the main components of snake venoms [66]. HDP-1 and HDP-2 are PLA2s that are isolated from V. nikolskii and could inhibit SARS-CoV-2 binding to ACE2 [67]. So, they can be effective in improving COVID-19 [68].

\section{Discussion}

Coronavirus pandemic induced by SARS-CoV-2 has been the cause of high burden worldwide. On 4 February 2022, the WHO announced 383,510,000 confirmed coronavirus illness 2019 cases and 5,700,000 deaths due to COVID-19. Although wide research has been carried out on the finding of effective therapeutics, so far there are no approved treatments against COVID-19. In the present study, harnessing natural toxic metabolites have been prioritized to make a review focusing on the efficacy of these metabolites as therapeutic agents [69]. Chloroquine/hydroxyl chloroquine combined with azithromycin had been used to improve COVID-19, but the results were controversial [70]. Remdesivir was the only approved antiviral drug for COVID-19 that was administrated for severe patients [71]. Some evidence indicates that toxins, toxoids, and venoms might be effective against SARS-CoV-2 through their antiinflammatory, $\mathrm{M}^{\text {Pro }}$ inhibitory, or antiviral effect. Due to the presence of melittin and phospholipase A2 (PLA2) in BV, it has antiviral effects on some enveloped and nonenveloped viruses. We know that after curing COVID-19, also some long-term effects may remain. BV has also a positive effect on recovery from long-COVID-19 [1]. Another idea is that fewer infected beekeepers are due to their less contact with other people and not for the effect of BV [3]. A low dose of pure botulinum toxin can reduce some symptoms of 
COVID-19-like acute respiratory failure and dyspnoea [14]. Tetanus toxin is similar to SARS-CoV-2 spike proteins and has been demonstrated that there is a correlation between DTP vaccination and the severity of COVID-19. Also, the rate of COVID-19 infection in pregnant women who received the TDaP vaccine decreases [30]. CRM197 that is a modified diphtheria toxin is similar to SARS-CoV-2 proteins and is present in some vaccines such as Hib and rubella. So, these vaccines may also have a protective effect on COVID-19 [34]. Cobrotoxin regulates the expression of genes involved in the inflammatory response and can prevent cytokines storm that causes more lung damage. Also, CD4/CD8 ratio that is imbalanced in COVID-19 can restore by cobrotoxin $[16,35]$. Tetrodotoxin inhibits main protease $\left(\mathrm{M}^{\text {Pro }}\right)$ and affects the replication of the virus [17]. Toxins, toxoids, and venoms may have effects on SARS-CoV-2 disease and prevent disease or reduce its symptoms. In addition, to achieve definitive results and efficacy of natural toxic metabolites comprehensive studies are needed (the data summary is presented in Table 1).

\section{Conclusion}

In this study, we investigated the role of toxins, toxoids, and venom on the pathophysiology of COVID-19 as an inflammatory disease. We discussed BV, botulinum toxin, tetanus toxin or toxoid, diphtheria toxin or modified toxin, cobrotoxin, and tetrodotoxin, which can be impressive in the pathophysiology of COVID-19. More surveys and clinical assessments are needed for better knowledge about the effect of toxins on COVID-19.

\section{Data Availability}

The data that appeared in this study are already publicly available in the literature.

\section{Ethical Approval}

This study was approved by the Ethics Committee of Hamadan University of Medical Sciences (IR.UMSHA.REC. 1400.441).

\section{Conflicts of Interest}

The authors declared no potential conflicts of interest.

\section{Authors' Contributions}

Mohammad Taheri and Fatemeh Nouri contributed equally to this work.

\section{Acknowledgments}

The authors are thankful to the Hamadan University of Medical Sciences for the financial support. This work was supported financially by Hamadan University of Medical Sciences, Hamadan, Iran (grant no. 140008046326).

\section{References}

[1] K. I. Kasozi, G. Niedbała, M. Alqarni et al., "Bee venom-a potential complementary medicine candidate for SARS-CoV2 infections," Frontiers in Public Health, vol. 8, p. 755, 2020.

[2] M. A. Erickson, E. M. Rhea, R. C. Knopp, and W. A. Banks, "Interactions of SARS-CoV-2 with the blood-brain barrier," International Journal of Molecular Sciences, vol. 22, no. 5, 2021.

[3] H. Männle, J. Hübner, and K. Münstedt, "Beekeepers who tolerate bee stings are not protected against SARS-CoV-2 infections," Toxicon: Official Journal of the International Society on Toxinology, vol. 187, pp. 279-284, 2020.

[4] C. Huang, Y. Wang, X. Li et al., "Clinical features of patients infected with 2019 novel coronavirus in Wuhan, China," Lancet, vol. 395, no. 10223, pp. 497-506, 2020.

[5] V. Izda, M. A. Jeffries, and A. H. Sawalha, "COVID-19: a review of therapeutic strategies and vaccine candidates," Clinical Immunology, vol. 222, Article ID 108634, 2021.

[6] M. Taheri, A. Bahrami, P. Habibi, and F. Nouri, A Review on the Serum Electrolytes and Trace Elements Role in the Pathophysiology of COVID-19. Biological Trace Element Research, Humana Press Inc., Totowa, NJ, USA, 2020.

[7] A. Bahrami, M. R. Arabestani, M. Taheri et al., "Exploring the role of heavy metals and their derivatives on the pathophysiology of COVID-19," Biological Trace Element Research, pp. 1-12, 2021.

[8] A. Shapira and I. Benhar, "Toxin-based therapeutic approaches," Toxins, vol. 2, no. 11, pp. 2519-2583, 2010.

[9] I. Kerkis, A. R. de Brandão Prieto da Silva, C. Pompeia, J. Tytgat, and P. L. de Sá Junior, "Toxin bioportides: exploring toxin biological activity and multifunctionality," Cellular and Molecular Life Sciences, vol. 74, no. 4, pp. 647-661, 2017.

[10] G. F. King, "Venoms as a platform for human drugs: translating toxins into therapeutics," Expert Opinion on Biological Therapy, vol. 11, no. 11, pp. 1469-1484, 2011.

[11] A. Schmidtko, J. Lötsch, R. Freynhagen, and G. Geisslinger, "Ziconotide for treatment of severe chronic pain," Lancet, vol. 375, no. 9725, pp. 1569-1577, 2010.

[12] V. O. Zambelli, K. F. M. Pasqualoto, G. Picolo, A. M. Chudzinski-Tavassi, and Y. Cury, "Harnessing the knowledge of animal toxins to generate drugs," Pharmacological Research, vol. 112, pp. 30-36, 2016.

[13] R. S. Norton, "Enhancing the therapeutic potential of peptide toxins," Expert Opinion on Drug Discovery, vol. 12, no. 6, pp. 611-623, 2017.

[14] M. Kandasamy, "Perspectives for the use of therapeutic Botulinum toxin as a multifaceted candidate drug to attenuate COVID-19," Medicine in Drug Discovery, vol. 6, Article ID $100042,2020$.

[15] G. Ietto, "Sars - CoV-2: reasons of epidemiology of severe ill disease cases and therapeutic approach using trivalent vaccine (tetanus, diphtheria and Bordetella pertussis)," Medical Hypotheses, vol. 141, Article ID 109779, 2020.

[16] F. Lin, P. F. Reid, and Z.-h. Qin, "Cobrotoxin could be an effective therapeutic for COVID-19," Acta Pharmacologica Sinica, vol. 41, no. 9, pp. 1258-1260, 2020.

[17] W. Y. Law, M. R. Asaruddin, S. A. Bhawani, and S. Mohamad, "Pharmacophore modelling of vanillin derivatives, favipiravir, chloroquine, hydroxychloroquine, monolaurin and tetrodotoxin as MPro inhibitors of severe acute respiratory syndrome coronavirus-2 (SARS-CoV-2)," BMC Research Notes, vol. 13, no. 1, p. 527, 2020. 
[18] W. Yang, F.-1. Hu, and X.-f. Xu, "Bee venom and SARS-CoV2," Toxicon, vol. 181, pp. 69-70, 2020.

[19] S. Bogdanov, Biological and Therapeutic Properties of Bee Venom, The Bee Venom Book, Muehlethurnen, Switzerland, 2016.

[20] Y. Al Naggar, J. P. Giesy, M. M. Abdel-Daim, M. J. Ansari, S. N. Al-Kahtani, and G. Yahya, "Fighting against the second wave of COVID-19: can honeybee products help protect against the pandemic?" Saudi Journal of Biological Sciences, vol. 28,2020

[21] I. Caramalho, A. Melo, E. Pedro et al., "Bee venom enhances the differentiation of human regulatory T cells," Allergy, vol. 70, no. 10, pp. 1340-1345, 2015.

[22] W. G. Lima, J. C. M. Brito, and W. S. Cruz Nizer, "Bee products as a source of promising therapeutic and chemoprophylaxis strategies against COVID - 19 (SARS-CoV -2)," Phytotherapy Research, vol. 35, no. 2, pp. 743-750, 2021.

[23] J. Block, High Risk COVID-19: Potential Intervention at Multiple Points in the COVID-19 Disease Process via Prophylactic Treatment with Azithromycin or Bee Derived Products, Preprints.org, Basel, Switzerland, 2020.

[24] U. Muthane and D. Panikar, "Botulinum toxins: pharmacology and its current therapeutic evidence for use," Neurology India, vol. 51, no. 4, pp. 455-460, 2003.

[25] P. Nigam and A. Nigam, "Botulinum toxin," Indian Journal of Dermatology, vol. 55, no. 1, pp. 8-14, 2010.

[26] S. S. Arnon, R. Schechter, T. V. Inglesby et al., "Botulinum toxin as a biological weapon," JAMA, vol. 285, no. 8, pp. 1059-1070, 2001.

[27] Z. F. Dembek, L. A. Smith, and J. M. Rusnak, "Botulism: cause, effects, diagnosis, clinical and laboratory identification, and treatment modalities," Disaster Medicine and Public Health Preparedness, vol. 1, no. 2, pp. 122-134, 2007.

[28] Z. Feng, Q. Sun, L. He et al., "Optimal dosage of botulinum toxin type A for treatment of glabellar frown lines," Dermatologic Surgery, vol. 41, no. 1, pp. S56-S63, 2015.

[29] D. Batifol, P. J. Finiels, S. Galmiche, P. Jammet, and M. de Boutray, "Could treatment with botulinum toxin protect against subsequent infection with COVID-19?" Journal of Stomatol Oral Maxillofac Surgey, 2021.

[30] C. D. Rickett, K. J. Maschhoff, and S. R. Sukumar, "Does tetanus vaccination contribute to reduced severity of the COVID-19 infection?" Medical Hypotheses, vol. 146, Article ID 110395, 2021.

[31] H. Sadeghi, Z. Robati, and M. J. Saharkhiz, "Variability in Zataria multiflora Bioss. essential oil of twelve populations from Fars province, Iran," Industrial Crops and Products, vol. 67, pp. 221-226, 2015.

[32] M. F. Nagoor Meeran, H. Javed, H. Al Taee, S. Azimullah, and S. K. Ojha, "Pharmacological properties and molecular mechanisms of thymol: prospects for its therapeutic potential and pharmaceutical development," Frontiers in Pharmacology, vol. 8, p. 380, 2017.

[33] P. A. Reche, "Potential cross-reactive immunity to SARSCoV-2 from common human pathogens and vaccines," Frontiers in Immunology, vol. 11, Article ID 586984, 2020.

[34] C. Pawlowski, A. Puranik, H. Bandi et al., "Exploratory analysis of immunization records highlights decreased SARSCoV-2 rates in individuals with recent non-COVID-19 vaccinations," Scientific Reports, vol. 11, no. 1, Article ID 4741, 2021.

[35] A. Ganji, I. Farahani, B. Khansarinejad, A. Ghazavi, and G. Mosayebi, "Increased expression of CD8 marker on T-cells in COVID-19 patients," Blood Cells, Molecules, and Diseases, vol. 83, Article ID 102437, 2020.

[36] V. Bane, M. Lehane, M. Dikshit, A. O’Riordan, and A. Furey, "Tetrodotoxin: chemistry, toxicity, source, distribution and detection," Toxins, vol. 6, no. 2, pp. 693-755, 2014.

[37] V. C. C. Cheng, S. K. P. Lau, P. C. Y. Woo, and K. Y. Yuen, "Severe acute respiratory syndrome coronavirus as an agent of emerging and reemerging infection," Clinical Microbiology Reviews, vol. 20, no. 4, pp. 660-694, 2007.

[38] X. Xue, H. Yu, H. Yang et al., "Severe acute respiratory syndrome coronavirus as an agent of emerging and reemerging infection," Journal of Virology, vol. 82, no. 5, pp. 2515-2527, 2008.

[39] D. Georgieva, R. K. Arni, and C. Betzel, "Proteome analysis of snake venom toxins: pharmacological insights," Expert Review of Proteomics, vol. 5, no. 6, pp. 787-797, 2008.

[40] J. J. Calvete, P. Juárez, and L. Sanz, "Snake venomics. Strategy and applications," Journal of Mass Spectrometry, vol. 42, no. 11, pp. 1405-1414, 2007.

[41] V. D. M. Muller, R. R. Russo, A. C. Oliveira Cintra et al., "Crotoxin and phospholipases A2 from Crotalus durissus terrificus showed antiviral activity against dengue and yellow fever viruses," Toxicon, vol. 59, no. 4, pp. 507-515, 2012.

[42] G. Borkow and M. Ovadia, "Selective lysis of virus-infected cells by cobra snake cytotoxins: a sendai virus, human erythrocytes, and cytotoxin model," Biochemical and Biophysical Research Communications, vol. 264, no. 1, pp. 63-68, 1999.

[43] R. Meenakshisundaram, S. Sweni, and P. Thirumalaikolundusubramanian, "Hypothesis of snake and insect venoms against Human Immunodeficiency Virus: a review," AIDS Research and Therapy, vol. 6, p. 25, 2009.

[44] T. Tasoulis and G. K. Isbister, "A review and database of snake venom proteomes," Toxins, vol. 9, no. 9, 2017.

[45] J. Slagboom, J. Kool, R. A. Harrison, and N. R. Casewell, "Haemotoxic snake venoms: their functional activity, impact on snakebite victims and pharmaceutical promise," British Journal of Haematology, vol. 177, no. 6, pp. 947-959, 2017.

[46] M. Ameziani, F. Chérifi, H. Kiheli et al., "Isolation and functional identification of an antiplatelet RGD-containing disintegrin from cerastes cerastes venom," The Protein Journal, vol. 39, no. 5, pp. 574-590, 2020.

[47] S. Saoud, F. Chérifi, T. Benhassine, and F. Laraba-Djebari, "Purification and characterization of a platelet aggregation inhibitor and anticoagulant Cc 5_NTase, CD 73-like, from Cerastes cerastes venom," Journal of Biochemical and Molecular Toxicology, vol. 31, no. 5, 2017.

[48] A. Munawar, S. Ali, A. Akrem, and C. Betzel, "Snake venom peptides: tools of biodiscovery," Toxins, vol. 10, no. 11, p. 474, 2018.

[49] A. C. M. Camargo, D. Ianzer, J. R. Guerreiro, and S. M. T. Serrano, "Bradykinin-potentiating peptides: beyond captopril,” Toxicon, vol. 59, no. 4, pp. 516-523, 2012.

[50] D. M. Lopes, N. E. G. Junior, P. P. C. Costa et al., "A new structurally atypical bradykinin-potentiating peptide isolated from Crotalus durissus cascavella venom (South American rattlesnake)," Toxicon, vol. 90, pp. 36-44, 2014.

[51] C. W. Chi, S. Z. Wang, L. G. Xu, M. Y. Wang, S. S. Lo, and W. D. Huang, "Structure-function studies on the bradykinin potentiating peptide from Chinese snake venom (Agkistrodon halys Pallas)," Peptides, vol. 6, no. 3, pp. 339-342, 1985.

[52] D. Gurwitz, "Angiotensin receptor blockers as tentative SARS-CoV-2 therapeutics," Drug Development Research, vol. 81, no. 5, pp. 537-540, 2020. 
[53] M. Hoffmann, H. Kleine-Weber, S. Schroeder et al., "SARSCoV-2 cell entry depends on ACE2 and TMPRSS2 and is blocked by a clinically proven protease inhibitor," Cell, vol. 181, no. 2, pp. 271-280, 2020.

[54] R. Lu, X. Zhao, J. Li et al., "Genomic characterisation and epidemiology of 2019 novel coronavirus: implications for virus origins and receptor binding," The Lancet, vol. 395, no. 10224 , pp. 565-574, 2020.

[55] F. L. van de Veerdonk, M. G. Netea, M. van Deuren et al., "Kallikrein-kinin blockade in patients with COVID-19 to prevent acute respiratory distress syndrome," Elife, vol. 9, 2020.

[56] A. S. Gouda and B. Mégarbane, "Snake venom-derived bradykinin-potentiating peptides: a promising therapy for COVID -19?" Drug Development Research, vol. 82, no. 1, pp. 38-48, 2021.

[57] L.-s. Chang, C. Chung, H.-B. Huang, and S.-r. Lin, "Purification and characterization of a chymotrypsin inhibitor from the venom of Ophiophagus hannah (King Cobra)," Biochemical and Biophysical Research Communications, vol. 283, no. 4, pp. 862-867, 2001.

[58] S. Seyedpour, B. Khodaei, A. H. Loghman et al., "Targeted therapy strategies against SARS-CoV-2 cell entry mechanisms: a systematic review of in vitro and in vivo studies," Journal of Cellular Physiology, vol. 236, no. 4, pp. 2364-2392, 2021.

[59] F. Chérifi and F. Laraba-Djebari, "Bioactive molecules derived from snake venoms with therapeutic potential for the treatment of thrombo-cardiovascular disorders associated with COVID-19," The Protein Journal, vol. 40, no. 6, pp. 799-841, 2021.

[60] N. Tang, D. Li, X. Wang, and Z. Sun, “Abnormal coagulation parameters are associated with poor prognosis in patients with novel coronavirus pneumonia," Journal of Thrombosis and Haemostasis, vol. 18, no. 4, pp. 844-847, 2020.

[61] M. Levi, J. Thachil, T. Iba, and J. H. Levy, "Coagulation abnormalities and thrombosis in patients with COVID-19," The Lancet Haematology, vol. 7, no. 6, pp. e438-e440, 2020.

[62] J. D. McFadyen, H. Stevens, and K. Peter, "The emerging threat of (Micro) Thrombosis in COVID-19 and its therapeutic implications," Circulation Research, vol. 127, no. 4, pp. 571-587, 2020.

[63] J. E. Gómez-Mesa, S. Galindo-Coral, M. C. Montes, and A. J. Muñoz Martin, "Thrombosis and coagulopathy in COVID-19," Current Problems in Cardiology, vol. 46, no. 3, Article ID 100742, 2021.

[64] T. Matsui, Y. Fujimura, and K. Titani, "Snake venom proteases affecting hemostasis and thrombosis," Biochimica et Biophysica Acta, vol. 1477, no. 1-2, pp. 146-156, 2000.

[65] Y. Yamazaki and T. Morita, "Snake venom components affecting blood coagulation and the vascular system:structural similarities and marked diversity," Current Pharmaceutical Design, vol. 13, no. 28, pp. 2872-2886, 2007.

[66] J. M. Gutiérrez and B. Lomonte, "Phospholipases A2: unveiling the secrets of a functionally versatile group of snake venom toxins," Toxicon: Official Journal of the International Society on Toxinology, vol. 62, pp. 27-39, 2013.

[67] A. E. Siniavin, M. A. Streltsova, M. A. Nikiforova et al., "Snake venom phospholipase A2s exhibit strong virucidal activity against SARS-CoV-2 and inhibit the viral spike glycoprotein interaction with ACE2," Cellular and Molecular Life Sciences, vol. 78, no. 23, pp. 7777-7794, 2021.

[68] B. Kalita, A. J. Saviola, S. P. Samuel, and A. K. Mukherjee, "State-of-the-art review - a review on snake venom-derived antithrombotics: potential therapeutics for COVID-19-associated thrombosis?" International Journal of Biological Macromolecules, vol. 192, pp. 1040-1057, 2021.

[69] D. Shokri and M. Rabbani khorasgani, "Evaluation of carbapenems resistance and frequency of Klebsiella pneumoniae carbapenemase (KPC) enzyme in Klebsiella pneumoniae strains isolated from clinical samples and determination of their acquired resistant profiles," Journal of Ilam University of Medical Sciences, vol. 24, no. 3, pp. 18-30, 2016.

[70] K. E. da Silva, T. R. Varella, G. M. d. S. Bet et al., "High mortality rate associated with KPC-producing Enterobacter cloacae in a Brazilian hospital," American Journal of Infection Control, vol. 46, no. 1, pp. 108-110, 2018.

[71] P. Tarighi, S. Eftekhari, M. Chizari, M. Sabernavaei, D. Jafari, and P. Mirzabeigi, "A review of potential suggested drugs for coronavirus disease (COVID-19) treatment," European Journal of Pharmacology, vol. 895, Article ID 173890, 2021. 